\title{
Extracorporeal Membrane Oxygenation Support for Adult Post-Cardiotomy Cardiogenic Shock
}

Wen-Je Ko, MD, Ching-Yuang Lin*, MD, Robert J. Chen, MD,

Shoei-Shen Wang, MD, Fang-Yue Lin, MD, Yih-Sharng Chen, MD

Running head title: ECMO for post-cardiotomy cardiogenic shock

From the Department of Surgery, National Taiwan University Hospital; and the Department of Pediatrics, Taipei Veteran General Hospital*, Taipei, Taiwan

Key words: extracorporeal membrane oxygenation, post-cardiotomy cardiogenic shock, intraaortic balloon pumping, ventricular assist device, myocardial stunning

Word count: 4851

Address reprint request to: Dr. Yih-Sharng Chen, Department of Surgery, National Taiwan University Hospital, 7, Chung-Shan S. Road, Taipei, Taiwan 100

Tel: 886-2-23123456 ext 3098

FAX: 886-2-23958747

E-mail:wenje@ha.mc.ntu.edu.tw

No competing interests declared 


\begin{abstract}
Background. Post-cardiotomy cardiogenic shock (PCS) often inflicts post-cardiac surgical patients. We report our experiences of using extracorporeal membrane oxygenation (ECMO) in adult patients with PCS, and analyze the factors that affected outcomes for these ECMO patients.
\end{abstract}

Methods. Retrospective review of medical records of the ECMO patients

Results. From Aug. 1994 to May 2000, 76 adult patients (48 male, 28 female; mean age:56.8 \pm 15.9 years) received ECMO support for PCS at the National Taiwan University Hospital. The mean ECMO blood flow was $2.53 \pm 0.84 \mathrm{~L} / \mathrm{min}$. The cardiac operations included coronary artery bypass grafting $(n=37)$, coronary artery bypass grafting and valvular surgery $(n=6)$, valvular surgery alone $(n=14)$, heart transplantation $(n=12)$, correction of congenital heart defects $(n=3)$, implantation of a left ventricular assist device $(n=2)$, and aortic operations $(n=2)$. Fifty-four patients received ECMO support after intra-aortic balloon pumping, but 22 patients directly received ECMO support.

Two patients were bridged to heart transplantation and two bridged to ventricular assist devices. Thirty patients died on ECMO support. The mortality etiologies included brain death $(n=3)$, refractory arrhythmia $(n=2)$, near motionless heart $(n=2)$, acute graft rejection $(n=1)$, primary graft failure $(n=1)$, uncontrolled bleeding $(n=5)$, and multiple organ failure $(n=16)$. Twenty-two patients were weaned off ECMO support but presented intrahospital mortality. The mortality etiologies included brain death $(n=1)$, sudden death 
$(n=4)$, and multiple organ failure $(n=17)$. Twenty patients were weaned off ECMO support and survived to hospital discharge. During the follow-up of $33 \pm 22$ months, all were in NYHA functional status I or II except two cases of late deaths. Among the ECMO-weaned patients, "dialysis for acute renal failure" was a significant factor in reducing the chance of survival.

Conclusions: ECMO provided a satisfactory partial cardiopulmonary support to patients with PCS, and allowed time for clinicians to assess patients and make an appropriate decision.

Abstract word count: 289 


\section{Introduction}

Post-cardiotomy cardiogenic shock (PCS) often inflicts post-cardiac surgical patients [1]. At least $1 \%$ of all post-cardiac surgical patients require mechanical circulatory support (MCS) beyond the conventional medical treatments to support the failing heart. Early results of extracorporeal membrane oxygenation (ECMO) treatment for PCS were very poor [2]. However, with the newly developed heparin-bound membrane oxygenator, better cannular design, centrifugal pump, and biocompatible bypass circuit, ECMO has reappeared as a new promising treatment for PCS [3]. The heparin-sparing effect is the most important advantage of new ECMO support for post-cardiotomy patients, because the risk of bleeding is high during the early post-cardiac surgical period. This study aims to report our experience of using ECMO to treat adult patients with refractory PCS, and to analyze the factors that affected the outcomes for these ECMO patients. 


\section{Patients and Methods}

ECMO had been used as an MCS at the National Taiwan University Hospital since Aug. 1994. Between Aug. 1994 and May 2000, 2912 adults ( $\geq 16$ years old) underwent open-heart operations at the hospital. The operations included coronary bypass grafting $(C A B G)(n=1318)$, heart valvular operations $(n=1194)$, and others $(n=400$, including correction of congenital heart diseases, heart transplantation, aortic operations, etc.). Beating-heart CABG began at the hospital in Feb. 2000, but the number was negligible during the period of data collection. The Intra-aortic balloon pump (IABP) was the first choice and ECMO support was the second, whenever MCS was required for PCS.

The ECMO system (catalog No. CB2505, Medtronic Inc., Anaheim, CA) consisted of a centrifugal pump and a hollow fiber microporous membrane oxygenator with an integrated heat exchanger. The whole ECMO circuit had a heparin-bound Carmeda-Bioactive surface. For simplicity the ECMO circuit was primed only with normal saline containing heparin at a concentration of $2 \mathrm{U} / \mathrm{mL}$. A further additional 5000 units of heparin were injected intravenously during cannulation. Heparin was not again used in the first 24 hours of ECMO support. For patients bridged to ECMO support directly from cardiopulmonary bypass (CPB), one half the regular dose of protamine was used, and heparin was not added to the priming solution. After 24 hours of ECMO support, heparin infusion was started to keep the activated clotting time in the range of 160-180 seconds, depending on a clinical judgment of the risk of bleeding. The dilution anemia occurring after the patient was connected to the ECMO was corrected by packed red blood cell transfusion. The hematocrit was maintained at 30-35\%. Although lower hematocrit reduced 
blood-oxygen carrying capacity, a higher hematocrit increased the risk of clot formation in the ECMO. Platelets were transfused when the platelet count was less than $50 \times 10^{3} / \mathrm{mm}^{3}$, unless a bleeding complication was present.

The femoral route was preferred to the open sternotomy route for ECMO support because the presence of an open sternotomy wound increased risks of bleeding and infection, and made nursing care more difficult. The centrifugal pump was typically set at a speed of 2000 revolutions per minute. The blood flow would be more than $2 \mathrm{~L} / \mathrm{min}$. A pulse oximeter was used to monitor the patient's arterial blood oxygen saturation. MX-2 tri-optic measurement cell (Medtronic Inc., Anaheim, CA) was attached to the pre- and post-oxygenator circuit to continuously monitor blood oxygen saturation and hematocrit. Continuous monitoring by the pulse oximeter and the MX-2 monitor, made frequent blood gas examination unnecessary. Blood gas was therefore checked once a day. Hematology and blood biochemistry were checked once a day and when clinically indicated. Inotropes infusion was only slowly tapered following initiation of ECMO support to prevent left heart distension, because ECMO increased the afterload of the left ventricle. Arterial pulse pressure was measured to monitor the function of the left heart and was used as a guide to taper inotropes infusion. A flattened arterial pressure wave indicated left heart drainage. Sedation by infusion of midazolam and fentanyl was routinely used. Fluid balance was maintained by diuretics. A hemofilter (FH66, Gambro, Hechingen, Germany) was connected between the ECMO arterial and venous circuits for hemofiltration, if dialysis was required.

Neither Microporous membrane oxygenator nor centrifugal pump was intended for prolonged use. The indications of ECMO replacement included severe plasma leakage from 
the oxygenator, deterioration of the oxygenator gas exchange function, decreased ECMO blood flow, hemoglobinuria, etc. A policy of replacing the entire ECMO circuits rather than the centrifugal pump or the oxygenator alone was followed for safety and simplicity.

Weaning off ECMO support was usually not attempted in the first 48 hours. The criteria for ECMO weaning included $\mathrm{SvO}_{2} \geq 70 \%$, stable hemodynamics and inotropes score $\leq 10$ (see note on Table 1), and echocardiographic determination of the absence of tamponade, the absence of left heart distension, and a left ventricular ejection fraction $\geq$ $35 \%$. ECMO blood flow was slowed to $0.5 \mathrm{~L} / \mathrm{min}$ for a while and the vital signs was observed. If hemodynamics remained stable, ECMO was removed at beside under intravenous anesthesia, and the vessels were primarily repaired. When the ECMO was being weaned off, inotropes infusion and ventilator setting were increased as necessary.

Medical records of the ECMO patients were reviewed. Data regarding demography (age, sex, preoperative NYHA classification, preoperative LVEF), cardiac operation (operative procedure, aortic clamp time, total CPB time), pre-ECMO condition (resuscitation, inotropes score, blood lactate level), ECMO procedure (place, route, blood flow, duration), biochemical changes and complications following ECMO support ( $24 \mathrm{hr}$ lactate level, transfusion amounts, CK, GOT, bilirubin, dialysis for acute renal failure) were recorded.

Data were analyzed by non-parametric methods. A significant difference was defined as $p$-value $<0.05$. The variables of groups of patients with different outcomes were compared using Fisher's exact test for categorical variables and Kruskal-Wallis test for continuous variables. Where the groups of patients differed, every pair of groups were 
further compared; again Fisher's exact test for categorical and Mann-Whitney U test for continuous variables. The statistical software was SAS 8.0 (SAS institute Inc., Cary, NC, USA) 


\section{Results}

\section{Background data:}

Seventy-eight adult post-cardiotomy patients received ECMO support in their early postoperative period. The indications were acute respiratory distress syndrome $(n=2)$ and cardiogenic shock $(n=76)$. Only the patients receiving ECMO support for PCS were included in this study. Their mean age was $56.8 \pm 15.9$ years. Forty-eight $(63 \%)$ patients were male, and 28 were female. Their operations included CABG $(n=37), C A B G$ and valvular operations $(n=6)$, valvular operation only $(n=14)$, heart transplantation $(n=12)$, operation for congenital heart defects $(n=3)$, implantation of a left ventricular assist device (VAD) $(n=2)$, and operation for dissecting aortic aneurysm $(n=2)$. The mean ECMO blood flow was $2.53 \pm 0.84 \mathrm{~L} / \mathrm{min}$. ECMO support was set up at the operation rooms for 39 patients and in the surgical intensive care units for 37 patients. Fifty-four patients had received IABP support before ECMO. Twenty-two patients (28.9\%) directly received ECMO support for the following reasons: 1) Extracorporeal cardiopulmonary resuscitation through open sternotomy wounds ( $n=7) .2)$ Right heart failure alone $(n=10)$. The cardiac operations of these 10 patients included tricuspid valve replacement for Ebstein anomaly (n $=2$ ), atrial septal defect closure in a patient with pulmonary hypertension, mitral valve replacement for rheumatic heart diseases $(n=3)$, heart transplantation $(n=2)$, and left VAD implantation $(n=2) .3)$. IABP contraindication $(n=5)$ because of low body weight $(\leq 30$ $\mathrm{kg})(\mathrm{n}=2)$, repair of dissecting aortic aneurysm $(\mathrm{n}=1)$, a modified Blalock-Taussig shunt in a patient with single ventricle $(n=1)$, and severe femoral artery atherosclerosis $(n=1)$.

The ECMO cannulation was via the right-atrium-aorta route $(n=15,19.7 \%)$, via the 
femoral vessels by the percutaneous Seldinger technique $(n=17,22.3 \%)$, or via the femoral vessels by the open method $(n=44,57.9 \%)$. Although we favored the femoral veno-arterial route, 15 patients received ECMO support through the open sternotomy wound. The femoral route was not selected, because 1) circulatory collapse occurred when the sternotomy wound was open, and emergent ECMO support was directly set up through the right-atrium-aorta route $(\mathrm{n}=6) ; 2)$ small body weight patients with small femoral vessels $(\mathrm{n}$ $=2)$; 3 ) femoral artery atherosclerosis was evident $(\mathrm{n}=1)$; 4) patients could not be weaned off $\mathrm{CPB}$, and were directly bridged to ECMO via the existing right atrium and aorta cannula $(n=3) ; 5)$ the renal graft on one side and IABP on the other side precluded the ECMO of the femoral route $(n=1) ; 6)$ left heart decompression by the left atrial drain was required $(n=2)$. Eight of these 15 sternotomy-ECMO patients directly received ECMO support without the prior IABP support.

\section{Outcome of ECMO support}

The patients were divided into 4 groups depending on their outcomes.

Bridge to heart transplantation $(n=2)$ and ventricular assist devices $(n=2)$ :

One heart recipient was put on the ECMO support directly from CPB because of primary graft failure; the patient was fortunate to get another donor heart 8 hours later. However, he died of a stroke 3 months after the heart retransplantation. One patient underwent emergent $\mathrm{CABG}$ for myocardial infarction complicated by cardiogenic shock, and needed ECMO support for failure to be weaned from CPB. After 31hours of ECMO support, he underwent successful heart transplantation, but he died suddenly 21 months thereafter. Another two patients used ECMO as a bridge to VAD. One patient underwent 
tricuspid valve replacement for Ebstein anomaly, but could not be weaned off CPB and was put on ECMO support. Finally, the ECMO was shifted to Thoratec right VAD after 117 hours of ECMO support. The patient underwent heart transplantation after 47 days of VAD support and was well at a 4-year follow-up. The other patient received open cardiac massage for persistent ventricular tachycardia/fibrillation on the first postoperative day of $\mathrm{CABG}$, and he was finally put on ECMO support with drainage from both atria. The ECMO shifted to centrifugal pump left VAD after 130 hours of support. The patient died of sepsis and multiple organ failure after he was on left VAD for a further 7 days.

\section{Death on the ECMO support $(n=30)$ :}

Thirty patients depended on the ECMO support until their deaths.

Brain death was noted in three CABG patients. Their ages were 56, 81, and 70 years, and their preoperative LVEF were 70,48 , and $42 \%$. Their failure to be weaned from CPB had no obvious causes. IABP followed by the femoral-ECMO was required to wean the patients from $\mathrm{CPB}$, but no resuscitation was performed during the operations. The patients did not wake after the operations, and brain death was noted on the first postoperative day. The ECMO supports were terminated at 31, 44, and 46 hours and the patients died. Although a head CT examination was not performed, intraoperative stroke was assumed to be the cause of the brain death. One patient had a refractory arrhythmia immediately after CABG operation. The patient died of the refractory ventricular arrhythmia 4 hours later, despite of the ECMO support. One patient had been stable on the femoral-ECMO for 187 hours but died of ventricular fibrillation possibly from a new myocardial infarction. Two patients had PCS with almost motionless hearts and died of circulatory shock even under 
ECMO support. One patient underwent heart retransplantation for chronic graft rejection; however, he required ECMO support for postoperative primary graft failure. The graft function recovered gradually under the ECMO support. However, two days later, while the patient remained on the ECMO support, ECG tracing revealed that QRS-wave widened and its amplitude decreased. The heart function deteriorated rapidly, and ECG tracing indicated cardiac standstill a few hours later. Necropsy revealed complement and IgG deposition on the vascular endothelium, and the final diagnosis was acute humoral rejection of the graft heart.

Five patients died of uncontrolled bleeding soon after the operations, while on ECMO support. Their ECMO durations were 3, 7, 12, 23, and 24 hours. One patient had primary graft failure and could not been weaned off CPB after the heart transplantation. She was put on ECMO support for 548 hours since a second donor heart was not available for heart retransplantation, and the ECMO was finally terminated at the family's request. The remaining 16 patients could not be weaned off the ECMO support, and eventually died of multiple organ failure while still on the ECMO support.

\section{Intrahospital mortality following wean off ECMO support $(\mathbf{n}=22)$ :}

A 67-year-old CABG patient suffered ventricular fibrillation while the sternotomy wound was being closed. ECMO support was applied after prolonged open cardiac message. Brain death was noted postoperatively, and ECMO support was removed at 69 hours. The patient survived for another three days before his cardiac standstill. Four patients suffered sudden death after ECMO had been removed for a while. Two of them were IABP dependent when ventricular fibrillation occurred; the other two patients had been removed 
from IABP support but remained bedridden. All four patients suffered no other organ dysfunction except persistent heart failure.

The remaining 17 patients were weaned off the ECMO support, but still died of multiple organ failure in the intensive care unit (ICU). The periods form ECMO removal to death were $24 \pm 49$ days, with a median of 8.2 days.

\section{Wean-off ECMO and survival to hospital discharge $(\mathrm{n}=20)$ :}

Twenty patients were weaned off ECMO support and survive to be discharged from the hospital. The intervals of ECMO support were $99 \pm 32$ hours. The intervals from ECMO removal to extubation and ICU discharge were $15 \pm 18$ days and $20 \pm 20$ days, respectively. These patients were followed up for $33 \pm 22$ months, and two late death occurred. One patient died suddenly at 11 months and the other died of pneumonia at 12 months. All other 18 patients are now in NYHA functional status II or I.

\section{Factors affecting outcomes of patients}

Figure 1 displays the distribution of ECMO durations of patients other than the four patients bridged to heart transplantation or VAD. Only two wean-and-survive patients required ECMO support beyond 6 days, actually 7 and 8 days. All survivors needed ECMO support for less than 8 days. Beyond 8 days, either patients were unlikely to recover from the post-cardiotomy heart failure or the ECMO complications would supervene and preclude the chance of survival.

Excluding patients with brain death, refractory arrhythmia, motionless heart, 
uncontrolled bleeding, etc., 16 patients died of multiple organ failure on ECMO support. Excluding one brain death and four sudden deaths, 17 patients were weaned off ECMO support but still died of multiple organ failure. These two groups of patients were compared the group of wean-and-survive patients. (Table 1) Although three groups presented decreasing trends in inotropes scores, ECMO blood flow, ECMO durations, blood lactate levels before ECMO support, blood lactate levels 24 hours after initiation of ECMO support, blood transfusion requirements, and peak GOT levels during the first 3 days, and peak total bilirubin levels during the first week after initiation of ECMO support, the decreases were not significantly different among them. Only "dialysis for acute renal failure" and "peak CK" significantly differed among the three groups of patients. The variables were compared between wean-but-die group and wean-and-survive group to find independent predictors of mortality in ECMO-weaned patients. Logistic regression analysis of the probability of mortality was conducted using the stepwise selection method. The stepwise logistic regression revealed "dialysis for acute renal failure" to be a significant predictor of mortality (odds ratio $=8,95 \%$ confidence interval: $1.4 \sim 45.7$ ). Acute renal failure, that occurred during the ECMO support and required dialysis, would reduce the chance of survival of those ECMO-weaned patients.

\section{Outcomes and complications of ECMO support via different routes}

The patients were divided into 3 groups depending on how the ECMO support was set up. (Table 2)

The chance of survival to discharge was the same for all three groups. Underlying diseases rather than the types of ECMO support decided the outcomes. The sternotomy 
group required the most transfusion, especially in the first day of the ECMO support. Four patients receiving the open-femoral EMCO support were found brain-dead after the operations. The etiologies were assumed to be intraoperative stroke in three patients and prolonged resuscitation after the operation in one patient. Two patients suffered severe hypoxic encephalopathy. The femoral arterial cannula of one patient accidentally slipped out, causing hypovolemic shock from severe bleeding. The other patient underwent prolonged resuscitation during the percutaneous transluminal coronary angioplasty before emergent CABG. All six patients with brain death or hypoxic encephalopathy eventually died. Three patients in the open-femoral ECMO group suffered hemiplegia/hemiparesis after the operations. No evidence indicated that these neurological complications resulted from ECMO support. No neurological complications were found in patients, who received percutaneous-femoral ECMO or sternotomy-ECMO support.

Inserting a reperfusion catheter into the distal femoral artery was not routine, and was performed according to clinical judgment. It was finally required in 16 patients who had received open-femoral-ECMO support and in four patients who had received percutaneous femoral-ECMO support. Toe cyanosis was noted in 5, 2, and 3 patients from each group, but the risk was the same among the three groups. Three patients required fasciotomy for the leg compartment syndrome caused by ischemia reperfusion injury after the insertion of a reperfusion catheter. Earlier insertion of the reperfusion catheter might have prevented this complication. 


\section{Comment}

When MCS was indicated, IABP was the first consideration because of its relative non-invasiveness. IABP decreases the left ventricular afterload and augments the coronary arterial perfusion. IABP is particularly beneficial for heart failure from ischemic heart diseases. However, IABP cannot remarkably increase cardiac output. Its effect is limited or infeasible in patients with profound heart failure, tachyarrhythmia, small body weight, or the right heart failure alone.

Successful use of HeartMate VAD [4] and Thoratec VAD [5] for PCS has been reported. However, due to technical complexity, these VADs are not suitable for critical patients in emergencies. Most institutions refuse to administer this expensive therapy in patients with only a moderate chance of survival, because of high cost of the VAD support. Strict criteria of patient selection deprived many critical patients of the opportunity to receive VAD support. A centrifugal pump can provide a cheaper and simpler VAD, but its use requires sternotomy. Left atrial drainage is not so easy [6]. Biventricular failure was common in patients with PCS $[7,8]$, and biventricular assistance was necessary in up to $50 \%$ of post-cardiotomy patients who required MCS [9]. Bilateral centrifugal pump support was complex and predisposed to lung edema [6].

In comparison, ECMO is a better MCS choice for PCS not amenable to IABP support alone. ECMO support has several advantages. ECMO composed of microporous membrane oxygenator and centrifugal pump allows rapid priming. ECMO support via the femoral route can be emergently set up at bedside under local anesthesia. ECMO support can be easily applied through the cannula also used for CPB. ECMO support can support both the 
right and left heart failure, and substitute the lung function. All these advantages make ECMO support an ideal means of cardiopulmonary rescue for critical patients with an uncertain diagnosis. ECMO is much cheaper than the currently available pulsatile VAD, and can provide a temporary support giving clinicians time to decide whether patients can benefit from further aggressive treatment. Four patients suffered brain death from intraoperative stroke or resuscitation, and one patient suffered hypoxic encephalopathy from preoperative resuscitation. Applying expensive VAD in these patients would be a waste. Myocardial stunning from ischemic-reperfusion injury is the most important etiology of PCS [10]. In theory, patients can recover from myocardial stunning regardless of the severity of cardiac dysfunction. However, no feasible methods distinguish irreversible infraction from reversible stunning in the immediate postoperative period. Seventeen patients were weaned off ECMO support, but later died of multiple organ failure. Multiple organ failure was attributed to severe secondary organs damage that had occurred before initiation of ECMO support. The severity of secondary organ damage was unknown, when MCS was used for PCS. ECMO support allowed time for medical decision. Then, if necessary, the patients could be bridged to heart transplantation or other more permanent VAD. The indication of ECMO support could be less strict, because of its relative simplicity and a lower cost. More patients could be evaluated and the next step decided upon. Patients could recover rapidly from myocardial stunning caused by ischemia reperfusion injury. Therefore, VAD, which could provide long-term MCS, was usually unnecessary for most PCS. Nearly 50\% (25/51) of Thoratec-VAD-supported post-cardiotomy survivors were supported for more than seven days, and the mean duration of support was 12.8 days [11]. Major operations involved in VAD implantation might delay 
patients' subsequent weaning-off VAD. In comparison, mean durations of ECMO support for post-cardiotomy survivors were $37.3 \pm 12.7$ hours in Magovern's report [6], $55.6 \pm 26.2$ hours in Muehrcke's report [12], and $99 \pm 33$ hours in this report. The ECMO support could be easily weaned off and removed; this may explain why ECMO support durations for post-cardiotomy survivors were much shorter than those of the VAD patients.

However, the effectiveness of ECMO support was limited in post-cardiotomy patients. ECMO can provide only partial cardiopulmonary support; $2.53 \pm 0.84 \mathrm{~L} / \mathrm{min}$ blood flow in this series. Bilateral VAD or total artificial heart is a better choice of MCS for patients with very poor heart function. ECMO support was not a solution to uncontrolled bleeding, and reliable hemostasis was a pre-requirement of successful ECMO support. ECMO support was not intended for long-term MCS as a bridge to heart transplantation. Complications usually developed during prolonged ECMO support that precluded heart transplantation [13]. Two of our patients were successfully bridged to heart transplantation after 8 and 31 hours of ECMO support, respectively. This was an exception not a rule. If a donor heart was not immediately available, patients should be bridged to VAD for long-term support. Four patients suffered sudden cardiac death some time after ECMO removal. Two remained IABP-dependent, and the other two were in NYHA functional status IV. Ventricular arrhythmia should be carefully prevented in ECMO-weaned patients with compromised heart function. Seventeen ECMO patients were weaned off the ECMO support, but later died of multiple organs failure. These wean-but-die patients had higher inotropes scores and blood lactate levels before the ECMO support, and higher CK, bilirubin, and GOT levels in the first week of ECMO support than did the wean-and-survive patients. The 
finding shows that wean-but-die patients had more severe shock damage before ECMO support than did the wean-and-survive patients. Earlier ECMO use might have saved some of them.

Kawahito et al. reported that ECMO support for PCS with a mean ECMO blood flow of $2.2 \pm 0.5 \mathrm{~L} / \mathrm{min}$ and mean support time of $27.4 \pm 26.7$ hours yielded $77 \%$ weaning and $39 \%$ long-term survival [14]. Fiser et al reported 31\% weaning and $16 \%$ survival [15]. ELSO registry revealed a 37\% survival rate in patients receiving ECMO for PCS [16]. ECMO support for PCS in this study yielded 58\% weaning and $28 \%$ survival to hospital discharge, if patients bridged to VAD or heart transplantation were excluded. Better case selection to exclude patients with uncontrollable bleeding, very poor cardiac contractility, and refractory ventricular arrhythmia, would have improved the result. Applying ECMO earlier, when it was indicated, would have decreased shock damage and prevented some patients dying of multiple organ failure.

Bleeding was the most important complication in ECMO support for PCS [6]. Heparin-bound Carmeda bioactive surface allowed an ECMO support with minimal or no systemic heparinization [17]. Lazzara et al. used the same heparin-bound ECMO support in post-cardiotomy patients; however, the mean packed red blood cells transfusion requirements were $24 \pm 9$ units for a mean support duration of 47.9 hours [18]. All of these patients received ECMO support through open sternotomy wounds. In contrast, most of our patients received ECMO support through the femoral route, and their blood transfusion requirements were much less, especially when the percutaneous insertion technique was employed. (See Table 2) ECMO support through the open sternotomy wounds provided 
higher blood flow due to the larger size of cannula. However, the ECMO blood flow was only marginally increased over that for the ECMO through the femoral route. The femoral-ECMO had fewer bleeding complications than the sternotomy-ECMO and allowed a much easier nursing care. Magovern et al [6] reported that two patients suffered strokes after the removal of ECMO support due to a clot on the tip of the cannula. A thromboembolism would go to legs rather than to the brain, if the femoral route were chosen for ECMO support. 


\section{Conclusion}

IABP is the first consideration, if MCS is indicated for PCS. ECMO is the second choice, when IABP support is insufficient or infeasible. ECMO support through the femoral route is preferred because of fewer related complications and easier nursing care. ECMO support allows a reasonable time to evaluate the patients and decide the next step, and prevents the misuse of expensive VAD.

\section{Acknowledgment}

This study was supported by grant NSC 90-2314-B002-428 from National Science Council, Taiwan. 


\section{References}

1. Downing TP, Miller DC, Stofer R, Shumway NE. Use of the intra-aortic balloon pump after valve replacement. J Thorac Cardiovasc Surg 1986;92:210-7

2. Jurmann J, Haverich A, Demertzis S, et al. Extracorporeal membrane oxygenation (ECMO): Extended indications for artificial support of both heart and lungs. Int J Artif Organs 1991;14:771-4

3. Anderson H, III, Steimle C, Shapiro M, et al. Extracorporeal life support for adult cardiorespiratory failure. Surgery 1993;114:161-73

4. DeRose JJ, Jr, Umana JP, Argenziano M, et al. Improved results for postcardiotomy cardiogenic shock with the use of implantable left ventricular assist devices. Ann Thorac Surg 1997;64:1757-62

5. Korfer R, El-Banayosy A, Posival H, et al. Mechanical circulatory support with the Thoratec assist device in patients with postcardiotomy cardiogenic shock. Ann Thorac Surg 1996;61:314-6

6. Magovern GJ, Jr, Magovern JA, Benckart DH, et al. Extracorporeal membrane oxygenation: Preliminary results in patients with postcardiotomy cardiogenic shock. Ann Thorac Surg 1994;57:1462-71

7. McBride LR, Naunheim KS, Fiore AC, Moroney DA, Swartz MT. Clinical experience with 111 Thoratec ventricular assist devices. Ann Thorac Surg $1999 ; 67: 1233-9$

8. Korfer R, El-Banayosy A, Arusoglu L, et al. Single-center experience with the Thoratec ventricular assist device. J Thorac Cardiovasc Surg 2000;119:596-600 
9. Pennington DG, Kanter KR, McBride LR, et al. Seven year's experience with the Pierce-Donarchy ventricular assist device. J Thorac Cardiovasc Surg 1988;96:901-11

10. Kloner RA, Przyklenk K, Kay GL. Clinical evidence for stunned myocardium after coronary artery bypass surgery. J Card Surg 1994;9:397-402

11. Thoratec VAD voluntary registry results. Thoratec Laboratories' Newsletter $1999 ; 14: 2-3$

12. Muehrcke DD, McCarthy PM, Stewart RW, et al. Extracorporeal membrane oxygenation for postcardiotomy cardiogenic shock. Ann Thorac Surg 1996;61:684-91

13. Delius RE, Zwischenberger JB, Cilley R, et al. Prolonged extracorporeal life support of pediatrc and adolescent cardiac transplant patients. Ann Thorac Surg $1990 ; 50: 791-5$

14. Kawahito K, Ino T, Adachi H, et al. Heparin coated percutaneous cardiopulmonary support for the treatment of circulatory collapse after cardiac surgery. ASAIO J $1994 ; 40: 972-6$

15. Fiser SM, Tribble CG, Kaza AK, et al. When to discontinue extracorporeal membrane oxygenation for postcardiotomy support. Ann Thorac Surg 2001;71:210-4

16. ECLS registry report. International summary. July, 2001.

17. Aranki SF, Adams DH, Rizzo RJ, et al. Femoral veno-arterial extracorporeal life support with minimal or no heparin. Ann Thorac Surg 1993;56:149-55

18. Lazzara RR, Magovern JA, Benckart DH, et al. Extracorporeal membrane oxygenation for adult post cardiotomy cardiogenic shock using a heparin bonded system. ASAIO J 1993;39:M444-7 
Table 1. Comparison of 3 groups of ECMO patients with different outcomes.

\begin{tabular}{|c|c|c|c|c|}
\hline & Die on ECMO & Wean but die & Wean and survive & $\mathrm{P}$ \\
\hline Patients Number & 16 & 17 & 20 & \\
\hline $\operatorname{Sex}(M / F)$ & $11 / 5$ & $13 / 4$ & $12 / 8$ & 0.536 \\
\hline Age (yr) & $54 \pm 17$ & $53 \pm 18$ & $54 \pm 16$ & 0.967 \\
\hline NYHA FC & & & & 0.391 \\
\hline I & 1 & 3 & 3 & \\
\hline II & 8 & 8 & 12 & \\
\hline III & 1 & 4 & 3 & \\
\hline IV & 6 & 2 & 2 & \\
\hline Op category & & & & 0.999 \\
\hline CABG & 7 & 7 & 10 & \\
\hline $\mathrm{CABG}+$ valve & 1 & 1 & 1 & \\
\hline CHD & 1 & 1 & 1 & \\
\hline HTX & 2 & 3 & 4 & \\
\hline Valve & 3 & 3 & 3 & \\
\hline Others & 2 & 2 & 1 & \\
\hline Site (OR/ICU) & $9 / 7$ & $7 / 10$ & $7 / 13$ & 0.448 \\
\hline CPR & 6 & 4 & 9 & 0.399 \\
\hline Dialysis for ARF & 12 & 11 & 5 & $0.006 *$ \\
\hline Pre-op LVEF & $47 \pm 20(n=11)$ & $48 \pm 18(n=13)$ & $54 \pm 19(\mathrm{n}=18)$ & 0.692 \\
\hline Aorta clamp time (min) & $117 \pm 29(n=14)$ & $117 \pm 38(\mathrm{n}=16)$ & $116 \pm 40(n=19)$ & 0.933 \\
\hline CPB time (min) & $227 \pm 115(n=16)$ & $191 \pm 70(n=18)$ & $190 \pm 88(\mathrm{n}=18)$ & 0.801 \\
\hline Inotrope scores & $86 \pm 96$ & $60 \pm 38$ & $51 \pm 40$ & 0.322 \\
\hline ECMO blood flow (L/min) & $2.78 \pm 0.75$ & $2.45 \pm 0.75$ & $2.36 \pm 0.62$ & 0.150 \\
\hline ECMO duration (h) & $132 \pm 139,89$ & $118 \pm 57,90$ & $99 \pm 33,96$ & 0.751 \\
\hline Lactate before ECMO (mmol/L) & $11.2 \pm 6.3$ & $11.2 \pm 6.2$ & $8.6 \pm 5.0$ & 0.428 \\
\hline Lactate $24 \mathrm{~h}$ after ECMO (mmol/L) & $7.2 \pm 5.3$ & $4.8 \pm 3.9$ & $4.2 \pm 4.1$ & 0.393 \\
\hline Blood transfusion (units) POD $1^{\#}$ & $6.4 \pm 5.2(\mathrm{n}=16)$ & $5.1 \pm 6.6(n=17)$ & $4.6 \pm 3.2(n=19)$ & 0.384 \\
\hline POD $2^{\#}$ & $4.9 \pm 6.7(n=15)$ & $3.6 \pm 4.2(n=17)$ & $2.0 \pm 1.5(n=19)$ & 0.493 \\
\hline Peak CK (U/L) & $5790 \pm 5287(15)$ & $13171 \pm 17487(17)$ & $4148 \pm 7409(20)$ & $0.027 *$ \\
\hline Peak GOT (U/L) & $1144 \pm 1589$ & $908 \pm 1339$ & $623 \pm 1335$ & 0.549 \\
\hline Peak total Bil. (mg/dL) & $13.0 \pm 14.3$ & $9.6 \pm 8.5$ & $6.8 \pm 5.8$ & 0.384 \\
\hline
\end{tabular}

\footnotetext{
* Comparison of categorical variables by Fisher's exact test, and continuous variables by
} 
Kruskal-Wallis test. "dialysis for ARF" is different between die-on-ECMO and wean-and-survive groups ( $\mathrm{p}=0.01$ ), and between wean-but-die and wean-and-survive groups ( $\mathrm{p}=0.02)$. "Peak CK" is different between wean-but-die and wean-and-survive groups $(\mathrm{p}=0.01)$

+ Inotrope score: dosages of dopamine + dobutamine $($ in $\mathrm{mcg} / \mathrm{kg} / \mathrm{min})+[$ dosages of epinephrine + norepinephrine + isoproterenol $($ in $\mathrm{mcg} / \mathrm{kg} / \mathrm{min})]$ x $100+$ dosages of milrinone (in $\mathrm{mcg} / \mathrm{kg} / \mathrm{min}$ ) x 15. "Inotrope score" here quantified the inotropes being infused when the ECMO was applied.

Peak CK, GOT were defined as the highest values noted in the first 3 days after initiation of ECMO support, and peak total Bil. the highest value in the first week after ECMO support.

ARF: acute renal failure; LVEF: left ventricular ejection fraction; NYHA FC: New York heart association function classification; CABG: coronary artery bypass grafting; CHD: congenital heart diseases; CK: creatine kinase; CPB: cardiopulmonary bypass; CPR: cardiopulmonary resuscitation; HTx: heart transplantation; GOT: glutamine oxalate transaminase. 
Table 2. Outcomes and complications of ECMO support delivered via different routes

\begin{tabular}{|c|c|c|c|c|}
\hline & Open femoral & $\begin{array}{c}\text { Percutaneous } \\
\text { femoral }\end{array}$ & Sternotomy & $\mathrm{P}$ \\
\hline Patients number & 44 & 17 & 15 & \\
\hline Arterial cannula $(\mathrm{Fr})$ & $17.3 \pm 1.2$ & $18.1 \pm 2.3$ & $18.3 \pm 2.5$ & 0.00 \\
\hline Venous cannula $(\mathrm{Fr})$ & $21.1 \pm 1.6$ & $20.9 \pm 1.4$ & $28.2 \pm 2.9$ & 0.00 \\
\hline ECMO blood flow (L/min) & $2.3 \pm 0.6$ & $2.6 \pm 1.0$ & $2.9 \pm 1.2$ & 0.75 \\
\hline \multicolumn{5}{|l|}{ Complications } \\
\hline \multicolumn{5}{|l|}{ Neurological: } \\
\hline Brain death & & 0 & 0 & \\
\hline Hypoxic encephalopathy & 4 & 0 & 0 & 0.00 \\
\hline Hemiplegia/hemiparesis & 2 & 0 & 0 & 0.00 \\
\hline Leg complication: & 3 & & & 0.00 \\
\hline Reperfusion catheter used & & 4 & 0 & 0.02 \\
\hline Toe cyanosis & 16 & 2 & 3 & 0.68 \\
\hline Fasciotomy & 5 & 0 & 0 & 0.31 \\
\hline Bleeding complications: & 3 & & & \\
\hline Transfusion $1^{\text {st }}$ day (units) & & $3.4 \pm 3.2(n=16)$ & $8.9 \pm 8.2(n=14)$ & 0.01 \\
\hline Transfusion $2^{\text {nd }}$ day (units) & $6.2 \pm 4.7(n=44)$ & $2.2 \pm 1.9(n=16)$ & $4.9 \pm 4.3(n=13)$ & 0.14 \\
\hline Resternotomy for bleeding/tamponade & $3.4 \pm 4.9(n=37)$ & 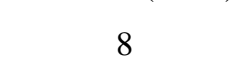 & 10 & 0.17 \\
\hline \multirow[t]{2}{*}{ Survival to hospital discharge } & 17 & 5 & 4 & 0.94 \\
\hline & 11 & & & \\
\hline
\end{tabular}

Note: 1. Kruskal-Wallis test for continuous variables. Fisher's exact test for categorical variables.

2. Only whole blood and packed red blood cells were included in the calculation of transfusion amount. 


\section{Figure Legends}

Figure 1. Distribution of ECMO durations in each group. Group I died on ECMO; Group II weaned off ECMO but die at hospital; Group III weaned off ECMO and survived to discharge from hospital. 
Ko-28
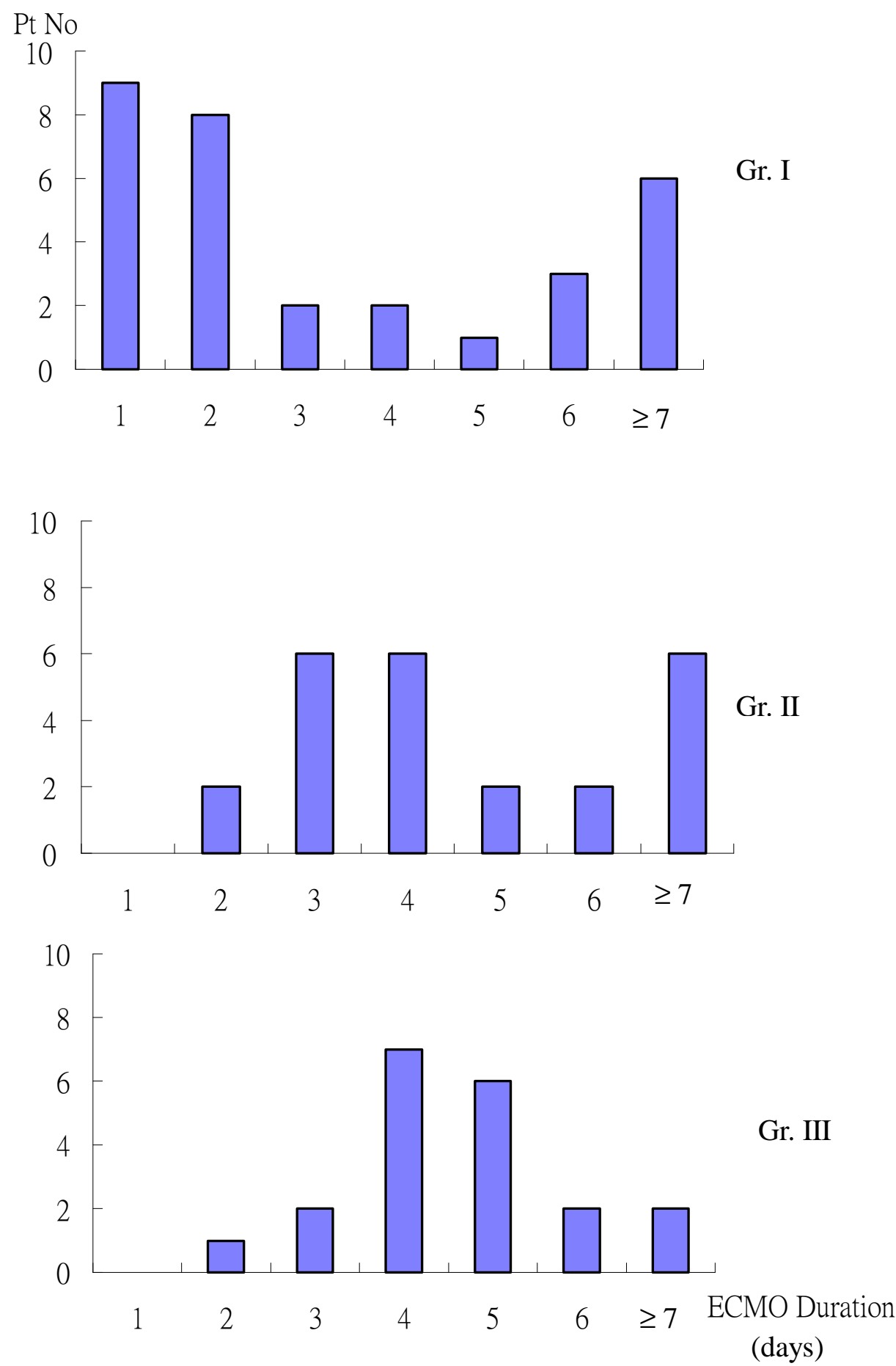

Fig 1 